\title{
Comprehensive histopathological comparison of epidermotropic/dermal metastatic melanoma and primary nodular melanoma
}

\author{
Stephanie L Skala, ${ }^{1}$ David P Arps, ${ }^{2}$ Lili Zhao, ${ }^{3}$ Kelly B Cha, ${ }^{4}$ Min Wang, ${ }^{1}$ Paul W Harms, ${ }^{1,4}$ \\ Aleodor A Andea, ${ }^{1,4}$ Douglas R Fullen ${ }^{1,4}$ \& May P Chan ${ }^{1,4}$ (D) \\ ${ }^{1}$ Department of Pathology, University of Michigan, Ann Arbor, MI, ${ }^{2}$ Consolidated Pathology Consultants, Libertyville, \\ IL, ${ }^{3}$ Department of Biostatistics, University of Michigan, and ${ }^{4}$ Department of Dermatology, University of Michigan, \\ Ann Arbor, MI, USA
}

Date of submission 28 May 2017

Accepted for publication 2 September 2017

Published online Article Accepted 7 September 2017

Skala S L, Arps D P, Zhao L, Cha K B, Wang M, Harms P W, Andea A A, Fullen D R \& Chan M P (2018) Histopathology 72, 472-480. https://doi.org/10.1111/his.13384

\section{Comprehensive histopathological comparison of epidermotropic/dermal metastatic melanoma and primary nodular melanoma}

Aims: Metastatic melanoma involving the epidermis and/or upper dermis may show significant histological overlap with primary cutaneous melanoma, especially the nodular subtype. Proper histopathological classification is crucial to appropriate staging and management, but is often challenging. The aim of this study was to identify helpful histopathological features for differentiating epidermotropic/dermal metastatic melanoma (EDMM) and primary nodular melanoma (PNM).

Methods and results: A cohort of EDMMs $(n=74)$ and PNMs $(n=75)$ was retrospectively reviewed for various histopathological features, and the data were compared between groups by the use of univariate analysis. Features significantly associated with EDMM included a tumour size of $<2 \mathrm{~mm}$, an absence of tumour-infiltrating lymphocytes and plasma cells, monomorphism, and involvement of adnexal epithelium. Features associated with PNM included a polypoid (exophytic) configuration, prominent tumour-infiltrating plasma cells (TIPs), a tumour size of $>10 \mathrm{~mm}$, ulceration, epidermal collarettes, a higher mitotic rate, necrosis, multiple phenotypes, significant pleomorphism, and lichenoid inflammation. In multivariate analysis, a logistic regression model including large tumour size, ulceration, prominent TIPs, lichenoid inflammation and epidermal collarettes was highly predictive of PNM. Six (8\%) EDMMs from three patients showed an 'epidermal-only' or 'epidermalpredominant' pattern closely simulating in-situ or microinvasive melanoma. Two of these cases were tested by fluorescence in-situ hybridisation, which confirmed clonal relationships with their corresponding primary melanomas.

Conclusions: This is the first comprehensive histopathological comparison of EDMM and PNM. Recognition of the above histopathological associations should aid in the correct classification and staging of cutaneous melanoma. Epidermotropic metastatic melanomas may occasionally show an epidermal-only/epidermal-predominant pattern; accurate diagnosis requires prudent clinical correlation and, when necessary, ancillary molecular tests.

Keywords: epidermotropic, metastatic melanoma, nodular melanoma, staging

Address for correspondence: May P Chan, MD, Department of Pathology, University of Michigan, 1301 Catherine Street, Medical Science I, M3261, Ann Arbor, MI 48109, USA. e-mail: mpchan@med.umich.edu 


\section{Introduction}

Distinguishing between metastatic and primary melanomas is crucial, as the therapy and prognosis differ widely. Whereas primary melanoma is treated with wide excision, metastatic melanoma is often treated with systemic therapy. ${ }^{1}$ Also, a patient with multiple primary melanomas is staged on the basis of the lesion with the worst prognostic parameters, while patients with satellite and in-transit metastases are considered to have stage IIIB disease, and those with distant cutaneous metastases are considered to have stage IV disease with a $10 \%$-year survival rate. ${ }^{2}$

Because the skin is a common site for melanoma metastasis, histological differentiation between primary and metastatic melanomas presents a rather common diagnostic dilemma. Primary nodular melanoma (PNM) typically only minimally involves the epidermis, frequently raising consideration for metastatic melanoma even in patients without a prior history of melanoma, as metastatic melanoma may develop following complete regression of the primary tumour. $^{3-5}$ Conversely, an epidermotropic/dermal metastatic melanoma (EDMM) may closely mimic a primary melanoma and be misdiagnosed as such, particularly when a prior history of melanoma is not known. Clinical correlation is often imperative in rendering the correct diagnosis. EDMM usually occurs near the site of a primary melanoma, sometimes presenting in crops. ${ }^{6}$ In contrast, it is rare for a patient to present with multiple synchronous primary melanomas. $^{7,8}$

Historically, epidermal involvement was considered to be pathognomonic for primary melanoma, ${ }^{9}$ but this belief was subsequently disputed. ${ }^{10}$ Microscopic features frequently reported in cutaneous metastatic melanoma included thinning of the epidermis, widening of the papillary dermis by aggregates of atypical melanocytes, flanking epidermal collarettes, a lack of inflammation, monomorphism, and fibrotic stroma. $^{10,11}$ Lymphovascular invasion was also thought to favour EDMM; however, more recent studies have demonstrated this feature in up to $30 \%$ of primary melanomas. ${ }^{12}$ Similarly, extension of intraepidermal melanocytes beyond dermal melanocytes (architectural 'shoulder') was once considered to be a unique feature of primary melanoma, but was later also reported in EDMM. ${ }^{8,13,14}$ The absence of an architectural shoulder in PNM further blurs their distinction.

Further complicating this issue are rare reports of purely intraepidermal epidermotropic metastatic melanoma. ${ }^{11,14,15}$ Despite their striking resemblance to melanoma in situ, the clinical presentation of numerous lesions and their small size, circumscription and symmetry supported the interpretation of epidermotropic metastatic melanomas with an 'epidermalonly' pattern.

In this study, we performed a comprehensive histopathological analysis of EDMM and PNM in order to identify the most helpful features for differentiating the two.

\section{Materials and methods}

This study has been approved by the Institutional Review Board at the University of Michigan. Our pathology database was searched for EDMM and PNM by the use of word searches for 'nodular' + 'melanoma', 'epidermotropic' + 'melanoma', and 'metastatic melanoma' from 2000 to 2016. Final classification as EDMM or PNM was based on careful clinicopathological correlation. Patients with prior history of melanoma were excluded from the PNM group, whereas patients without a previously diagnosed primary melanoma were excluded from the EDMM group. Metastases distant from the previous primary melanoma site were also excluded from the EDMM group; only locoregional metastases (e.g. from the same extremity) were included. To qualify as EDMM, a metastatic melanoma should involve the epidermis/adnexal epithelium and/or the upper dermis (papillary dermis or superficial reticular dermis). Haematoxylin and eosin (H\&E)-stained slides of the final cohort were reviewed by two dermatopathologists (D.P.A. and M.P.C.) and a pathology resident (S.L.S.) for various histopathological features, as listed in Tables 1 and 2. Selected features are defined below.

A tumour was polypoid (exophytic) if its epicentre was above the skin surface. An architectural shoulder was defined as an intraepidermal component extending at least three rete ridges beyond the dermal component. Pleomorphism was scored as follows: 0, mostly monomorphic cells; 1 , pleomorphism appreciated at medium to high magnification; and 2, pleomorphism readily appreciated at low magnification, often with bizarre-looking cells. Tumours consisting of two or more morphologically distinct cell populations were considered to have multiple phenotypes. Mitotic rate was the number of dermal mitotic figures in a $1-\mathrm{mm}^{2}$ 'hot spot' (i.e. most mitotically active area). As per the American Joint Committee on Cancer guidelines for melanoma, ${ }^{2}$ tumour-infiltrating lymphocytes (TILs) were either absent (0), non-brisk 
Table 1. Histopathological features showing statistically significant differences between epidermotropic/dermal metastatic melanoma (EDMM) and primary nodular melanoma (PNM) by univariate analysis

\begin{tabular}{|c|c|c|c|c|}
\hline Histopathological features & $\begin{array}{l}\text { EDMM } \\
(n=74)\end{array}$ & $\begin{array}{l}\text { PNM } \\
(n=75)\end{array}$ & $P$-value & $\begin{array}{l}\text { Specificity } \\
(\%)\end{array}$ \\
\hline Greatest diameter $<2$ mm, no. (\%) & $19(26)$ & $0(0)$ & $<0.0001$ & 100 \\
\hline $\begin{array}{l}\text { Absence of tumour-infiltrating } \\
\text { plasma cells, no. (\%) }\end{array}$ & $67(91)$ & $38(51)$ & 0.0036 & 49 \\
\hline Monomorphism, no. (\%) & $41(55)$ & $24(32)$ & 0.0309 & 68 \\
\hline $\begin{array}{l}\text { Absence of tumour-infiltrating } \\
\text { lymphocytes, no. (\%) }\end{array}$ & $9(12)$ & $2(3)$ & 0.0348 & 97 \\
\hline $\begin{array}{l}\text { Involvement of adnexal } \\
\text { epithelium, no. (\%) }\end{array}$ & $32(43)$ & $18(24)$ & 0.0417 & 76 \\
\hline $\begin{array}{l}\text { Polypoid (exophytic) } \\
\text { configuration, no. (\%) }\end{array}$ & $0(0)$ & $16(21)$ & $<0.0001$ & 100 \\
\hline $\begin{array}{l}\text { Prominent tumour-infiltrating } \\
\text { plasma cells, no. (\%) }\end{array}$ & $1(1)$ & $22(29)$ & $<0.0001$ & 99 \\
\hline $\begin{array}{l}\text { Greatest diameter }>10 \mathrm{~mm} \text {, } \\
\text { no. }(\%)\end{array}$ & $2(3)$ & $31(41)$ & $<0.0001$ & 97 \\
\hline Ulceration, no. (\%) & $4(5)$ & $36(48)$ & $<0.0001$ & 95 \\
\hline Epidermal collarettes, no. (\%) & $18(24)$ & $53(71)$ & $<0.0001$ & 76 \\
\hline Mean mitotic rate (median, range) & $4(2,0-23)$ & $13(11,1-53)$ & $<0.0001$ & - \\
\hline Tumour necrosis, no. (\%) & $2(3)$ & $19(25)$ & 0.0002 & 97 \\
\hline Multiple phenotypes, no. (\%) & $6(8)$ & $24(32)$ & 0.0012 & 92 \\
\hline Significant pleomorphism, no. (\%) & $13(18)$ & $31(41)$ & 0.0073 & 82 \\
\hline Lichenoid inflammation, no. (\%) & $3(4)$ & $14(19)$ & 0.0088 & 96 \\
\hline
\end{tabular}

(1), or brisk (2). Tumour-infiltrating plasma cells (TIPs) were scored as follows: 0 , no intratumoral plasma cells; 1, rare aggregates of intratumoral plasma cells only appreciable upon close inspection; and 2, prominent, readily appreciable aggregates of intratumoral plasma cells. Regression was defined as a discrete fibrotic area with melanophages, lymphocytes, and increased vascularity. Tumoral melanosis refers to large aggregates of melanophages replacing a portion of the melanoma. Unlike lymphovascular invasion, in which melanoma cells are present within a vascular lumen, angiotropism refers to extravascular melanoma cells bulging into the vascular lumen but covered by endothelial cells.

In univariate analysis (performed by M.P.C.), comparison of each feature between EDMM and PNM was performed with chi-square tests (categorical) or twosample $t$-tests (continuous). Multivariate analysis was conducted by L.Z. with sAS (version 9.4; SAS Institute, Cary, NC, USA). A logistic regression model was obtained by a stepwise variable selection procedure based on a significance level of 0.15 . The parameter estimates, $P$-values from Wald chi-square tests and the area under the receiver operating characteristic (ROC) curve were reported. A $P$-value of $<0.05$ was considered to be significant.

Fluorescence in-situ hybridisation (FISH) was performed by A.A.A., P.W.H. and M.W. on selected EDMM cases with an unusual 'epidermal-only' or 'epidermal-predominant' pattern and their corresponding primary tumours to confirm clonal relationships. Four-micrometre sections were prepared from each selected formalin-fixed paraffin-embedded tissue block; one was stained with H\&E, and two were hybridised by use of the Vysis Melanoma FISH probe kit, including probes 6p25 (RREB1), 6q23 (MYB), CEP6, and 11q13 (CCND1), and Vysis LSI probes 8q24 (MYC) and 9p21 (CDKN2A)/CEP9, according to the manufacturer's instructions. Thirty cells were evaluated, and the percentage of nuclei with copy 
Table 2. Histopathological features without statistically significant differences between primary nodular melanoma (PNM) and epidermotropic/dermal metastatic melanoma $(E D M M)$ by univariate analysis

\begin{tabular}{|c|c|c|c|}
\hline Histopathological features & $\begin{array}{l}\text { PNM } \\
(n=75) \\
\text { no. }(\%)\end{array}$ & $\begin{array}{l}\text { EDMM } \\
(n=74) \\
\text { no. }(\%)\end{array}$ & $P$-value \\
\hline Associated naevus & $8(11)$ & $2(3)$ & 0.0578 \\
\hline Perineural invasion & $12(16)$ & $5(7)$ & 0.0991 \\
\hline Symmetry* & $12 / 74(16)$ & 20/69 (29) & 0.1036 \\
\hline Infiltrative borders & $38(51)$ & $52(70)$ & 0.1238 \\
\hline Lymphovascular invasion & $18(24)$ & $10(14)$ & 0.1405 \\
\hline Regression & $13(17)$ & $7(9)$ & 0.1946 \\
\hline $\begin{array}{l}\text { Expansion of papillary } \\
\text { dermis by melanoma }\end{array}$ & $69(92)$ & $54(73)$ & 0.2004 \\
\hline Pseudomaturation & $15(20)$ & $9(12)$ & 0.2364 \\
\hline $\begin{array}{l}\text { Brisk tumour-infiltrating } \\
\text { lymphocytes }\end{array}$ & $8(11)$ & $5(7)$ & 0.4054 \\
\hline Tumoral melanosis & $14(19)$ & $10(14)$ & 0.4379 \\
\hline $\begin{array}{l}\text { Pseudoepitheliomatous } \\
\text { hyperplasia }\end{array}$ & $10(13)$ & $7(9)$ & 0.4970 \\
\hline Architectural 'shoulder' & $18(24)$ & $14(19)$ & 0.5017 \\
\hline Lentiginous growth & $57(76)$ & $62(84)$ & 0.5949 \\
\hline Pagetoid spread & $48(64)$ & $52(70)$ & 0.6455 \\
\hline Junctional nests & $55(73)$ & $51(69)$ & 0.7559 \\
\hline Sheet-like growth & $31(41)$ & $33(45)$ & 0.7642 \\
\hline Angiotropism & $11(15)$ & $10(14)$ & 0.8614 \\
\hline $\begin{array}{l}\text { Involvement of preserved } \\
\text { rete ridges }\end{array}$ & $48(64)$ & $49(66)$ & 0.8709 \\
\hline
\end{tabular}

* Some cases were partial biopsies in which symmetry could not be determined.

number changes, including gain of $6 \mathrm{p} 25$, loss of $6 \mathrm{q} 23$, gain of $11 \mathrm{q} 13$, gain of $8 \mathrm{q} 24$, and homozygous loss of $9 \mathrm{p} 21$, was recorded and considered as positive or negative on the basis of predetermined thresholds. $^{16}$

\section{Results}

The final cohort consisted of 74 EDMM specimens obtained from 44 patients, and 75 PNM specimens obtained from 75 patients. There was no overlap of the patient pools of the two groups. Epidermal
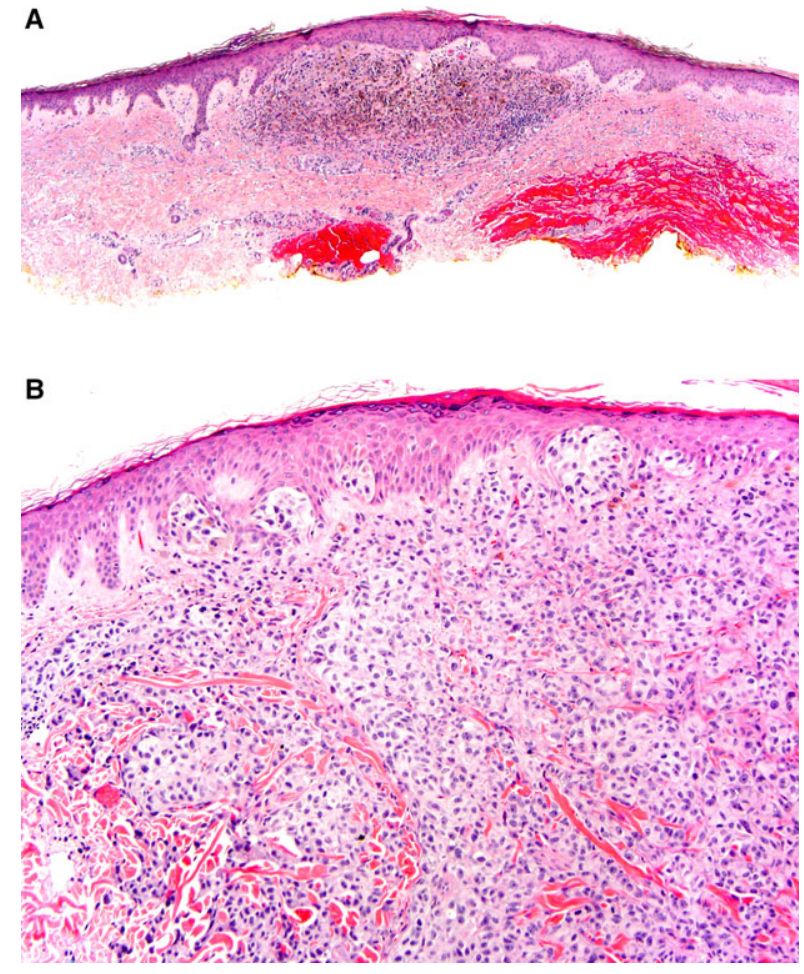

Figure 1. Representative features associated with epidermotropic/ dermal metastatic melanoma. A, A small lesion in the upper dermis measuring $<2 \mathrm{~mm}$ in greatest dimension [haematoxylin and eosin (H\&E)]. B, Another lesion shows a dermal nodule with epidermotropism, cytological monomorphism, and lack of tumour-infiltrating lymphocytes and plasma cells (H\&E).

involvement was observed in $62(84 \%)$ EDMM cases and $59(79 \%)$ PNM cases. Histopathological features showing statistically significant differences between EDMM and PNM by univariate analysis are listed in Table 1. Features associated with EDMM included a greatest dimension of $<2 \mathrm{~mm}$, a TIP score of 0 , a pleomorphism score of 0 , a TIL score of 0 , and involvement of the adnexal epithelium (Figure 1). Features associated with PNM included a polypoid (exophytic) configuration, a TIP score of 2, a greatest dimension of $>10 \mathrm{~mm}$, ulceration, epidermal collarettes, a higher mitotic rate, tumour necrosis, multiple phenotypes, a pleomorphism score of 2, and lichenoid inflammation (Figure 2). Ulceration was seen much more frequently in lesions $>10 \mathrm{~mm}(24 /$ 33; $73 \%)$ than in lesions $<10 \mathrm{~mm}(16 / 116 ; 14 \%)$ $(P<0.0001)$. Other examined features did not show significant differences between groups (Table 2). Multivariate analysis resulted in a predictive model for PNM that included large tumour size, ulceration, prominent TIPs, lichenoid inflammation, and epidermal collarettes (Table 3). The area under the ROC 


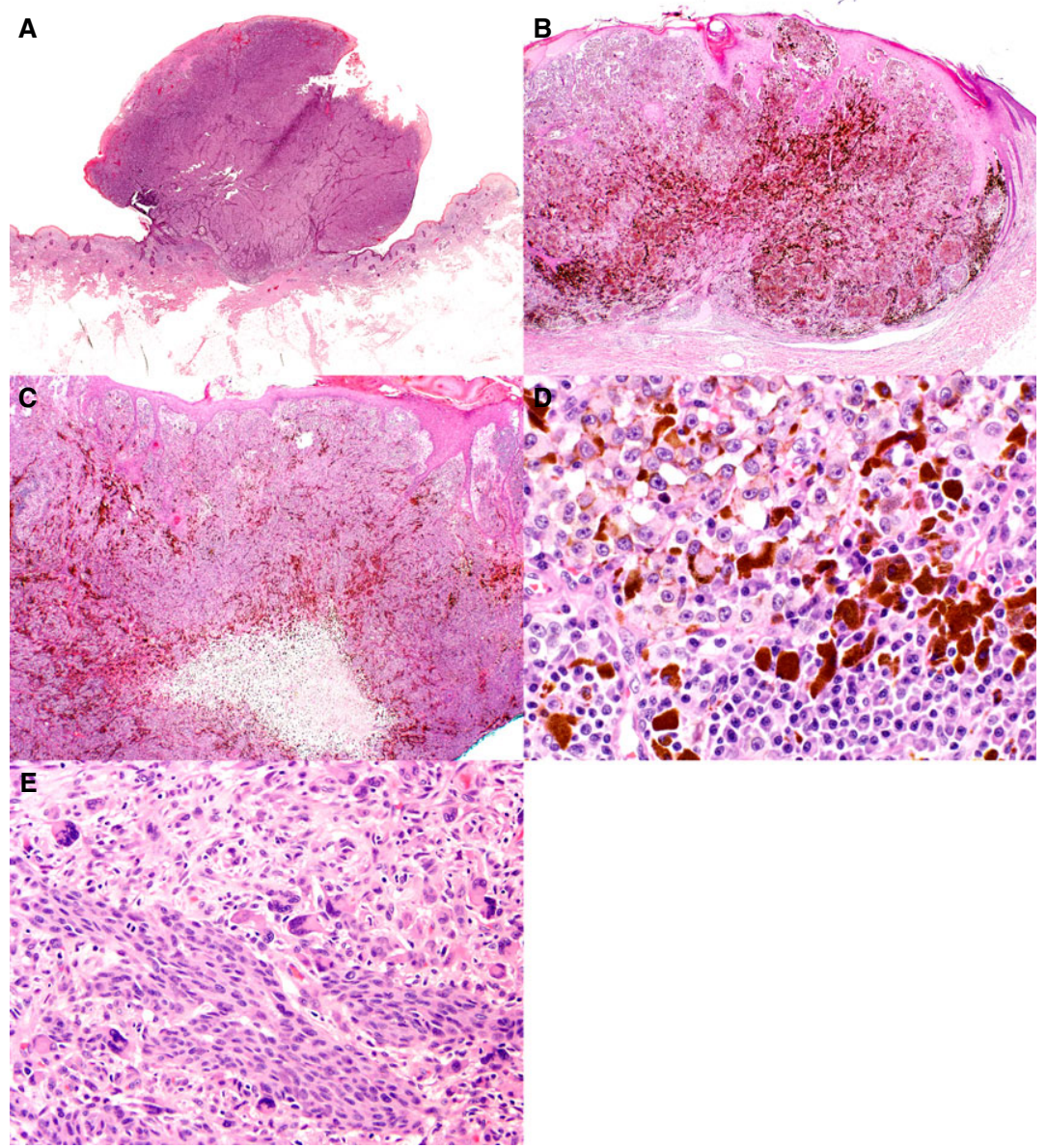

Figure 2. Representative features associated with primary nodular melanoma. A, A large, ulcerated nodular melanoma with an exophytic polypoid configuration [haematoxylin and eosin (H\&E)]. B, This tumour is flanked by epidermal collarettes (H\&E). C, Zonal tumour necrosis is noted in the deeper portion of this biopsy (H\&E). D, Clusters of tumourinfiltrating plasma cells are present in this example (H\&E). E, This nodular melanoma shows multiple distinct phenotypes and significant pleomorphism. One population of cells contains highly pleomorphic and bizarrelooking nuclei (top), whereas the other population consists of nests of relatively uniform cells (bottom) (H\&E).

Table 3. Logistic regression model by multivariate analysis

\begin{tabular}{lll}
\hline Histopathological features & $\begin{array}{l}\text { Estimates } \\
\text { with PNM }\end{array}$ & $P$-value \\
\hline $\begin{array}{l}\text { Greatest diameter of }>10 \mathrm{~mm} \\
(\text { versus }<2 \mathrm{~mm})\end{array}$ & 2.2024 & 0.0023 \\
\hline $\begin{array}{l}\text { Greatest diameter of } 2-10 \mathrm{~mm} \\
\text { (versus }<2 \mathrm{~mm})\end{array}$ & 0.5340 & 0.3695 \\
\hline $\begin{array}{l}\text { Presence of ulceration } \\
\text { Presence of prominent } \\
\text { tumour-infiltrating plasma cells }\end{array}$ & 0.8014 & 0.0166 \\
\hline Presence of lichenoid inflammation & 0.7993 & 0.0487 \\
\hline Presence of epidermal collarette & 0.6903 & 0.0675 \\
\hline
\end{tabular}

PNM, primary nodular melanoma.

*A greater positive estimate indicates a higher probability of PNM.

curve was 0.90 , indicating a high predictive accuracy of this model.

Six EDMM lesions from three patients were purely or predominantly intraepidermal, and were microscopically indistinguishable from melanoma in situ and microinvasive melanoma (Figure 3). In two of these patients, multiple new pigmented lesions developed around the site of a previously resected primary scalp melanoma. Another patient presented with multiple small pigmented lesions on the right leg after excision of a primary right heel melanoma. Two epidermal-only/epidermal-predominant EDMM lesions from the two patients with scalp melanoma were studied by FISH, which showed identical copy number changes to those in their corresponding primary melanomas (Table S1), confirming a clonal relationship.

\section{Discussion}

The histological distinction between cutaneous metastatic melanoma and primary melanoma has important diagnostic, prognostic and treatment implications, but, to our knowledge, none of the previous studies have systematically compared the histopathological features of the two. We selected 

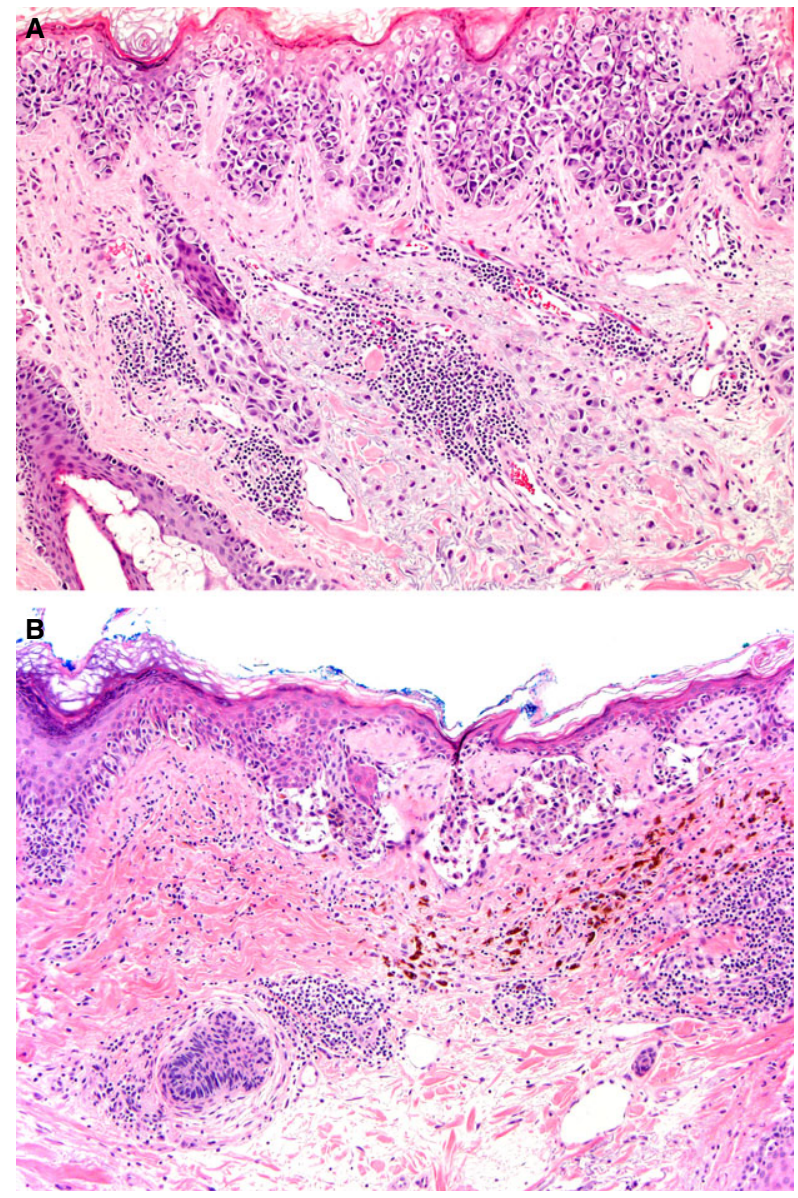

Figure 3. Epidermotropic metastatic melanomas with epidermalpredominant and epidermal-only patterns. A, This scalp lesion was taken from near the site of a previously resected primary melanoma. Most of the melanoma cells are present within the epidermis, giving rise to an architectural 'shoulder' and the appearance of a microinvasive melanoma. Adnexal involvement is also present [haematoxylin and eosin (H\&E)]. B, Another patient presented with a purely intraepidermal metastatic melanoma on the scalp, closely resembling melanoma in situ (H\&E). [Colour figure can be viewed at wileyonlinelibrary.com].

only metastatic melanoma involving the epidermis and/or upper dermis, because these cases most closely simulate primary melanoma. Likewise, we confined our cohort of primary melanoma to the nodular subtype, as it is most likely to generate diagnostic confusion with EDMM.

We identified multiple useful histopathological features for differentiating EDMM and PNM. Of these, tumour size is one of the most objective discriminating factors. Nodules $>10 \mathrm{~mm}$ are more likely to be primary, whereas lesions $<2 \mathrm{~mm}$ are much more likely to be metastatic. Because large melanomas are more likely to ulcerate, we also found PNM to be associated with ulceration. Although none of the
PNM lesions in our series was smaller than $2 \mathrm{~mm}$, one should be aware that primary 'micromelanomas' do exist, and may be detected more readily with the use of dermoscopy. ${ }^{17,18}$ Hence, tumour size should be viewed as a strong but not absolute discriminator of metastatic and primary melanomas.

In addition to tumour size, tumour silhouette also provides useful information. Polypoid/exophytic lesions are much more likely to be PNM, as only one EDMM in our series showed a polypoid silhouette. The rarity of polypoid EDMM is also reflected in the literature. ${ }^{11,19}$ Epidermal collarettes have been previously reported in both EDMM and primary melanomas. $^{20}$ In our cohort, epidermal collarettes were significantly associated with PNM. This association is probably related to the larger tumour size in this group, as epidermal collarettes result from expansile dermal growth.

None of the intraepidermal features (lentiginous growth, junctional nests, pagetoid spread, and involvement of preserved rete ridges) are associated with either group. Interestingly, involvement of the adnexal epithelium is significantly associated with EDMM, indicating that, in addition to epidermotropism, adnexotropism is also common in metastatic melanoma.

Although architectural shoulder was traditionally thought to be specific for primary melanoma, later studies have also shown this feature in EDMM. ${ }^{8,13,14}$ Our findings support the latter by showing architectural shoulder in $19 \%$ of EDMMs. Notably, six EDMM lesions from three patients were purely or predominantly intraepidermal, and were microscopically indistinguishable from melanoma in situ and microinvasive melanoma. The metastatic nature of these lesions is supported by the identical copy number abnormalities to those in their corresponding primary melanomas. The occurrence of multiple lesions in the vicinity of the primary melanoma site also provides compelling clinical evidence for metastases. However, when clinicopathological correlation fails to elucidate the primary versus metastatic nature of a lesion, molecular studies may be needed to facilitate more accurate staging. ${ }^{15,21}$

A number of cytomorphological features aid in the distinction between PNM and EDMM. In general, significant pleomorphism favours a diagnosis of PNM, whereas monomorphism favours a diagnosis of EDMM. Furthermore, primary melanomas are more likely to comprise multiple morphologically distinct subpopulations secondary to genetic divergence within the tumour. In contrast, metastatic melanomas more commonly show one phenotype only, 
probably reflective of a selected tumour subclone harbouring increased metastatic potential. ${ }^{22}$ Pseudomaturation, referring to partial diminution of cell size with dermal descent, has been described in the majority of naevoid melanomas ${ }^{23}$ but also in other melanoma subtypes, as well as EDMM. This feature is not helpful in their distinction.

Although a coexisting naevus tends to be found more frequently in PNM than in EDMM, the difference falls short of statistical significance $(P=0.0578)$. It is well known that primary melanoma may arise from a pre-existing naevus via malignant transformation. A recent study showed that the incidence of associated naevus was highest among superficial spreading melanomas (37\%) and lowest among nodular melanomas $(16 \%){ }^{24}$ This relatively low frequency in PNM may have accounted for the lack of a significant difference from EDMM.

Although mitotic figures can be brisk in metastatic melanoma, ${ }^{25}$ our study showed a significantly higher mitotic rate in PNM. Nevertheless, given the wide range and significant overlap of mitotic rates in the two groups, the observed difference is of limited practical value. As rapidly proliferating tumours often outgrow their blood supply, tumour necrosis may result, and is again more commonly found in PNM. Previous studies have found tumour necrosis to be significantly associated with increased tumour thickness, an increased Ki67 proliferation index, and ulceration. ${ }^{26,27}$ It is therefore possible that the lower incidence of tumour necrosis in EDMM may be, in part, attributable to smaller tumour size and infrequent ulceration in this group.

Melanoma can metastasise via lymphatic, haematogenous or angiotropic routes. ${ }^{28-33}$ Both angiotropism and lymphovascular invasion are fair predictors of metastatic potential in melanoma. ${ }^{34}$ In our study, both lymphovascular invasion and angiotropism were seen in small subsets of PNM and EDMM cases, and failed to distinguish between groups. Perineural invasion is believed to be another means by which satellite metastases form in melanoma. This, too, does not serve as a useful discriminator.

Our data indicate that prominent TIPs are strongly suggestive of PNM, being observed in 29\% of these cases as compared with 1\% of EDMMs. Assessment of TIPs is fairly straightforward, making it a powerful and practical discriminator. Mascaro et al. reported clusters of plasma cells in $22 \%$ of primary melanomas, a feature that was associated with increased Breslow thickness, ulceration, and poor survival. ${ }^{35} \mathrm{~A}$ recent study confirmed these associations, as well as higher mitotic activity. ${ }^{36}$ Interestingly, when found in metastatic melanomas, TIPs were reported to constitute a favourable prognosticator. ${ }^{37}$ Further investigation is needed to elucidate the immunological relationship between TIPs and melanoma cells.

Because absence of TILs is associated with sentinel lymph node metastasis, whereas brisk TILs are associated with prolonged recurrence-free survival, ${ }^{38,39}$ one may expect fewer TILs in metastatic melanomas. A study reported that a higher percentage of primary melanomas $(67 \%)$ contained lymphoid infiltrate than metastatic foci $(9 \%)$ in the same patients. ${ }^{40}$ Another study of nodal metastatic melanomas found that TILs were absent in $46 \%$ of cases. ${ }^{41}$ In the skin, we showed that absence of TILs is significantly associated with EDMM, suggesting an enhanced ability to evade immune detection. ${ }^{42}$ Similarly, lichenoid inflammation is less common in EDMM than in PNM, despite the essentially identical frequency of epidermal involvement in these groups.

Perhaps contrary to common belief, ${ }^{11}$ our study did not identify any significant difference in the frequency of tumour regression. Late regression, as evidenced by fibrosis, increased vascularity, epidermal atrophy, and melanophages, has been reported in $10-35 \%$ of primary melanomas. ${ }^{43}$ Although a review article cited a much lower incidence of spontaneous regression in metastatic melanomas $(0.25 \%)$, this figure is probably skewed by the exceedingly low incidence of regression in extracutaneous metastases. ${ }^{44}$ Our cohort showed regression in $17 \%$ and $9 \%$ of PNM and EDMM cases, respectively. Several factors may explain the lack of a significant difference. First, regression has been reported to occur less frequently in nodular melanoma (13\%) than in other subtypes. ${ }^{45}$ Second, the histopathological features of regression are not specific, and may be indistinguishable from changes of chronic friction or prior trauma. Finally, one of our EDMM cases with regression was excised after immunotherapy (pembrolizumab), which may have triggered regression of the metastatic melanoma. ${ }^{46}$ Tumoral melanosis - a histological variant of regression ${ }^{47}$ - similarly fails to differentiate between PNM and EDMM.

In conclusion, we conducted the first comprehensive histopathological comparison of EDMM and PNM, and identified multiple useful discriminators with significant associations that have not been previously reported. Of these, the collective findings of large tumour size, ulceration, prominent TIPs, epidermal collarettes and lichenoid inflammation strongly support a diagnosis of PNM on the basis of a logistic regression model. Other highly useful characteristics 
based on univariate analysis include small tumour size, and an absence of TILs, which favour EDMM, and a polypoid configuration, tumour necrosis, and multiple phenotypes, which favour PNM. Although the importance of clinical correlation cannot be overemphasised, recognition of the above constellation of histopathological features, especially when the clinical history is limited, should allow for more accurate classification, and hence more precise tumour staging and prognostication for melanoma patients.

\section{Conflicts of interest}

The authors declare no conflicts of interest.

\section{Author contributions}

S. L. Skala, D. P. Arps, D. R. Fullen and M. P. Chan designed the research study. S. L. Skala, D. P. Arps, K. B. Cha, M. Wang, P. W. Harms, A. A. Andea and M. P. Chan obtained the data. S. L. Skala, L. Zhao, M. Wang, P. W. Harms, A. A. Andea and M. P. Chan analysed the data. S. L. Skala and M. P. Chan wrote the paper. All authors performed critical review of the manuscript.

\section{References}

1. PDO Adult Treatment Editorial Board. Melanoma treatment $(P D Q)$ : health professional version. $P D Q$ cancer information summaries. Bethesda, MD: National Cancer Institute, 2017. https:// www.ncbi.nlm.nih.gov/books/NBK66034/; accessed 25 August 2017.

2. Balch CM, Gershenwald JE, Soong S-J et al. Final version of 2009 AJCC melanoma staging and classification. J. Clin. Oncol. 2009; 27; 6199-6206.

3. High WA, Stewart D, Wilbers CR et al. Completely regressed primary cutaneous malignant melanoma with nodal and/or visceral metastases: a report of 5 cases and assessment of the literature and diagnostic criteria. J. Am. Acad. Dermatol. 2005; 53; 89-100.

4. Emanuel PO, Mannion M, Phelps RG. Complete regression of primary malignant melanoma. Am. J. Dermatopathol. 2008; 30; $178-181$.

5. Mărgăritescu I, Chiriţă AD, Vasilescu F. Completely regressed primary cutaneous melanoma-difficulties in diagnosis and classification. Rom. J. Morphol. Embryol. 2014; 55 (2 Suppl.); 635-642.

6. Ariel IM. Disseminated melanoma with unique unilateral distribution. Cancer 1975; 36; 2143-2146.

7. Warner TF, Gilbert EF, Ramirez G. Epidermotropism in melanoma. J. Cutan. Pathol. 1980; 7; 50-54.

8. Bengoechea-Beeby MP, Velasco-Oses A, Mourino Fernandez F et al. Epidermotropic metastatic melanoma: are the current histologic criteria adequate to differentiate primary from metastatic melanoma? Cancer 1993; 72; 1909-1913.
9. Allen AC, Spitz S. Malignant melanoma: a clinicopathological analysis of the criteria for diagnosis and prognosis. Cancer 1953; 6; 1-45.

10. Kornberg R, Harris M, Ackerman AB. Epidermotropically metastatic malignant melanoma. Arch. Dermatol. 1978; 114; 6769.

11. Plaza JA, Torres-Cabala C, Evans H et al. Cutaneous metastases of malignant melanoma: a clinicopathologic study of 192 cases with emphasis on the morphologic spectrum. Am. J. Dermatopathol. 2010; 32; 129-136.

12. Storr SJ, Safuan S, Mitra A et al. Objective assessment of blood and lymphatic vessel invasion and association with macrophage infiltration in cutaneous melanoma. Mod. Pathol. 2011; 25; 493-504.

13. Heenan PJ, Clay CD. Epidermotropic metastatic melanoma simulating multiple primary melanomas. Am. J. Dermatopathol. 1991; 13; 396-402.

14. Abernethy JL, Soyer HP, Kerl H et al. Epidermotropic metastatic malignant melanoma simulating melanoma in situ: a report of 10 examples from two patients. Am. J. Surg. Pathol. 1994; 18; 1140-1149.

15. Lestre S, João A, Ponte $P$ et al. Intraepidermal epidermotropic metastatic melanoma: a clinical and histopathological mimicker of melanoma in situ occurring in multiplicity. J. Cutan. Pathol. 2011; 38; 514-520.

16. Gerami P, Jewell SS, Morrison LE et al. Fluorescence in situ hybridization (FISH) as an ancillary diagnostic tool in the diagnosis of melanoma. Am. J. Surg. Pathol. 2009; 33; 11461156.

17. Bono A, Bartoli C, Baldi M et al. Micro-melanoma detection. A clinical study on 22 cases of melanoma with a diameter equal to or less than $3 \mathrm{~mm}$. Tumori 2004; 90; 128-131.

18. Bono A, Tolomio E, Trincone S et al. Micro-melanoma detection: a clinical study on 206 consecutive cases of pigmented skin lesions with a diameter $\leq 3 \mathrm{~mm}$. Br. J. Dermatol. 2006; 155; 570-573.

19. Hayashi H, Kawashima T, Hosokawa K et al. Epidermotropic metastatic malignant melanoma with a pedunculated appearance. Clin. Exp. Dermatol. 2003; 28; 666-668.

20. Guerriere-Kovach PM, Hunt EL, Patterson JW et al. Primary melanoma of the skin and cutaneous melanomatous metastases: comparative histologic features and immunophenotypes. Am. J. Clin. Pathol. 2004; 122; 70-77.

21. Bahrami S, Cheng L, Wang M et al. Clonal relationships between epidermotropic metastatic melanomas and their primary lesions: a loss of heterozygosity and X-chromosome inactivation-based analysis. Mod. Pathol. 2007; 20; 821-827.

22. Nowell PC. The clonal evolution of tumor cell populations. Science 1976; 194; 23-28.

23. Zembowicz A, McCusker M, Chiarelli C et al. Morphological analysis of nevoid melanoma: a study of 20 cases with a review of the literature. Am. J. Dermatopathol. 2001; 23; 167-175.

24. Lin WM, Luo S, Muzikansky A et al. Outcome of patients with de novo versus nevus-associated melanoma. J. Am. Acad. Dermatol. 2015; 72; 54-58.

25. Bulkley GB, Cohen MH, Banks PM et al. Long-term spontaneous regression of malignant melanoma with visceral metastases: report of a case with immunologic profile. Cancer 1975; 36; 485-494.

26. Bachmann IM, Ladstein RG, Straume O et al. Tumor necrosis is associated with increased alpha(v)beta(3) integrin expression and poor prognosis in nodular cutaneous melanomas. BMC Cancer 2008; 8; 362. 
27. Ladstein RG, Bachmann IM, Straume O et al. Tumor necrosis is a prognostic factor in thick cutaneous melanoma. Am. J. Surg. Pathol. 2012; 36; 1477-1482.

28. Gerami P, Shea C, Stone MS. Angiotropism in epidermotropic metastatic melanoma: another clue to the diagnosis. Am. J. Dermatopathol. 2006; 28; 429-433.

29. Barnhill R, Lugassy C. Angiotropic malignant melanoma and extravascular migratory metastasis: description of 36 cases with emphasis on a new mechanism of tumour spread. Pathology 2004; 36; 485-490.

30. Johnson C, Ramos-Caro F, Hassanein A. Ultra-late erysipeloid angiotropic metastatic malignant melanoma. Int. J. Dermatol. 2001; 40; 446-447.

31. Moreno A, Espanol I, Romagosa V. Angiotropic malignant melanoma. Report of two cases. J. Cutan. Pathol. 1992; 19; 325329.

32. Saluja A, Money N, Zivony D et al. Angiotropic malignant melanoma: a rare pattern of local metastases. J. Am. Acad. Dermatol. 2001; 44; 829-832.

33. Shea C, Kline $\mathrm{M}$, Lugo $\mathrm{J}$ et al. Angiotropic metastatic malignant melanoma. Am. J. Dermatopathol. 1995; 17; 5862.

34. Barnhill R, Dy K, Lugassy C. Angiotropism in cutaneous melanoma: a prognostic factor strongly predicting risk for metastasis. J. Invest. Dermatol. 2002; 119; 705-706.

35. Mascaro JM, Molgo M, Castel T et al. Plasma cells within the infiltrate of primary cutaneous malignant melanoma of the skin: a confirmation of its histoprognostic value. Am. J. Dermatopathol. 1987; 9; 497-499.

36. Bosisio FM, Wilmott JS, Volders $\mathrm{N}$ et al. Plasma cells in primary melanoma. Prognostic significance and possible role of IgA. Mod. Pathol. 2016; 29; 347-358.

37. Erdag G, Schaefer JT, Smolkin ME et al. Immunotype and immunohistologic characteristics of tumor infiltrating immune cells are associated with clinical outcome in metastatic melanoma. Cancer Res. 2012; 72; 1070-1080.

38. Azimi F, Scolyer RA, Rumcheva $\mathrm{P}$ et al. Tumor-infiltrating lymphocyte grade is an independent predictor of sentinel lymph node status and survival in patients with cutaneous melanoma. J. Clin. Oncol. 2012; 30; 2678-2683.
39. Fortes C, Mastroeni S, Mannooranparampil TJ et al. Tumorinfiltrating lymphocytes predict cutaneous melanoma survival. Melanoma Res. 2015; 25; 306-311.

40. Payan HM, Gilbert EF, Jacobs WH. Lymphocytic reaction around primary and metastatic melanomas. South. Med. J. 1970; 63; 1350-1352.

41. Mihm MC Jr, Clemente CG, Cascinelli N. Tumor-infiltrating lymphocytes in lymph node melanoma metastases: a histopathologic prognostic indicator and an expression of local immune response. Lab. Invest. 1996; 74; 43-47.

42. Osborn JL, Greer SF. Metastatic melanoma cells evade immune detection by silencing STAT1. Int. J. Mol. Sci. 2015; 16; 4343-4361.

43. Blessing K, McLaren KM. Histological regression in primary cutaneous melanoma: recognition, prevalence and significance. Histopathology 1992; 20; 315-322.

44. Kalialis LV, Drzewiecki KT, Klyver H. Spontaneous regression of metastases from melanoma: a review of the literature. Melanoma Res. 2009; 19; 275-282.

45. Tas F, Erturk K. Presence of histological regression as a prognostic factor in cutaneous melanoma patients. Melanoma Res. 2016; 26; 492-496.

46. Brizio M, Fava P, Astrua C et al. Complete regression of melanoma skin metastases after electrochemotherapy plus ipilimumab treatment: an unusual clinical presentation. Eur. J. Dermatol. 2015; 25; 271-272.

47. Ng SH, Chave TA. Tumoral melanosis as a manifestation of a completely regressed primary melanoma with metastasis. $\mathrm{Br}$. J. Dermatol. 2006; 155; 627-628.

\section{Supporting Information}

Additional Supporting Information may be found in the online version of this article:

Table S1. Comparison of copy number changes detected by fluorescence in-situ hybridisation in two cases of epidermal-only/predominant metastatic melanoma with their corresponding primary tumours. 